\title{
On Routing in Random Rayleigh Fading Networks
}

\author{
Martin Haenggi, Senior Member, IEEE
}

\begin{abstract}
This paper addresses the routing problem for large wireless networks of randomly distributed nodes with Rayleigh fading channels. First, we establish that the distances between neighboring nodes in a Poisson point process follow a generalized Rayleigh distribution. Based on this result, it is then shown that, given an end-to-end packet delivery probability (as a quality of service requirement), the energy benefits of routing over many short hops are significantly smaller than for deterministic network models that are based on the geometric disk abstraction. If the permissible delay for short-hop routing and long-hop routing is the same, it turns out that routing over fewer but longer hops may even outperform nearest-neighbor routing, in particular for high end-to-end delivery probabilities.
\end{abstract}

Index Terms-Ad hoc networks, communication systems, fading channels, Poisson processes, probability, routing.

\section{INTRODUCTION}

$\mathbf{E}$ NERGY consumption in multihop wireless networks is a crucial issue that needs to be addressed at all the layers of the communication system, from the hardware up to the application. In this paper, we focus on routing strategies that employ hops of different length in a network whose nodes constitute a Poisson point process. The analysis is based on a Rayleigh fading channel model, and the results demonstrate that the properties of the physical channel have a substantial impact on optimum protocol design at the network layer.

Often, a deterministic "disk model" is used for the analysis of multihop packet networks [1]-[9], where the radius for a successful transmission has a deterministic value, irrespective of the condition of the wireless channel. Interference is taken into account using the same geometric disk abstraction. The stochastic nature of the fading channel and thus the fact that the signal-to-noise-and-interference ratio (SINR) is a random variable are neglected. Using such models, it is easy to show that, for a path loss exponent of $\alpha$, there is an energy gain of $n^{\alpha-1}$ if a hop over a distance $d$ is split into $n$ hops of distance $d / n$. However, the volatility of the channel cannot be ignored in wireless networks [10], [11]; the inaccuracy of "disk models" has also been pointed out in [12] and is easily demonstrated experimentally [13], [14]. In addition, the "prevalent all-or-nothing model" [15] leads to the assumption that a transmission over a multihop path either fails completely or is $100 \%$ successful, ignoring the fact that end-to-end packet

Manuscript received February 6, 2003; revised November 23, 2003; accepted June 1, 2004. The editor coordinating the review of this paper and approving it for publication is J. C. Hou. This work was supported in part by the DARPA/IXO-NEST Program (AF-F30602-01-2-0526), NSF (ECS02-25265 and ECS03-29766), and ORAU.

The author is with the Department of Electrical Engineering at the University of Notre Dame, Notre Dame, IN 46556 USA (e-mail: mhaenggi@nd.edu).

Digital Object Identifier 10.1109/TWC.2005.850376 loss probabilities increase with the number of hops (unless the transmit power is adapted).

To overcome some of these limitations of the "disk model," we employ a simple Rayleigh fading link model that relates transmit power, large-scale path loss, and the success of a transmission. The end-to-end packet delivery probability over a multihop route is the product of the link-level reception probabilities.

While fading has been considered in the context of packet networks [16], [17], its impact on the network (and higher) layers is largely an open problem. This paper attempts to shed some light on this cross-layer issue by analyzing the performance of different routing schemes under Rayleigh fading. In Section II, we introduce the link model and show that noise and interference can be treated separately. We also prove that the internode distances in a Poisson point process follow a generalized Rayleigh distribution; we define and determine the path efficiency; and we introduce the five routing strategies we consider. In Section III, the energy consumption of those five routing strategies is analyzed; Section IV discusses the performance of those routing schemes under equal delay constraints, and Section $\mathrm{V}$ concludes the paper.

\section{RAYleigh Network MOdel}

\section{A. Rayleigh Fading Link Model}

We assume a narrowband Rayleigh block fading channel. A transmission from node $i$ to node $j$ is successful if the SINR $\gamma_{i j}$ is above a certain threshold $\Theta$ that is determined by the communication hardware and the modulation and coding scheme [10]. The SINR $\gamma$ is a discrete random process given by

$$
\gamma=\frac{R}{N+I}
$$

$R$ is the received power, which is exponentially distributed with mean $\bar{R}$. Over a transmission of distance $d=\left\|x_{i}-x_{j}\right\|_{2}$ with an attenuation $d^{\alpha}$, we have $\bar{R}=P_{0} d^{-\alpha}$, where $P_{0}$ is proportional to the transmit power. ${ }^{1} N$ denotes the noise power, and $I$ is the interference power affecting the transmission, i.e., the sum of the received power from all the undesired transmitters.

Theorem 1: In a Rayleigh fading network, the reception probability $p_{\mathrm{r}}:=\mathbb{P}[\gamma \geqslant \Theta]$ can be factorized into the reception

\footnotetext{
${ }^{1}$ This equation does not hold for very small distances. So, a more accurate model would be $\bar{R}=P_{0}^{\prime} \cdot\left(d / d_{0}\right)^{-\alpha}$, valid for $d \geqslant d_{0}$, with $P_{0}^{\prime}$ as the average value at the reference point $d_{0}$, which should be in the far field of the transmit antenna. At $916 \mathrm{MHz}$, for example, the near field may extend up to $1 \mathrm{~m}$ (several wavelengths).
} 
probability of a zero-noise network and the reception probability of a zero-interference network.

Proof: Let $R_{0}$ denote the received power from the desired source and $R_{i}, i=1, \ldots, k$, the received power from $k$ interferers. All the received powers are exponentially distributed, i.e., $p_{R_{i}}\left(r_{i}\right)=1 / \bar{R}_{i} \exp \left(-r_{i} / \bar{R}_{i}\right)$, where $\bar{R}_{i}$ denotes the average received power $\bar{R}_{i}=P_{i} d_{i}^{-\alpha}$. The probability of correct reception is ${ }^{2}$

$$
\begin{aligned}
p_{\mathrm{r}} & =\mathbb{P}\left[R_{0} \geqslant \Theta(I+N)\right] \\
& =\mathbb{E}_{I}\left\{\operatorname { e x p } \left[-\frac{\Theta(I+N)]}{\left.\left.\bar{R}_{0}\right]\right\}}\right.\right. \\
& =\int_{0}^{\infty} \ldots \int_{0}^{\infty} \exp \left\{-\frac{\Theta\left[\sum_{i=1}^{k} r_{i}+N\right)}{\bar{R}_{0}}\right\} \\
& =\underbrace{\exp \left(-\frac{\Theta N}{P_{0} d_{0}^{-\alpha}}\right)}_{p_{\mathrm{r}}^{N}} \cdot \underbrace{\prod_{i=1}^{k} \frac{1}{1+\Theta \frac{P_{i}}{P_{0}}\left(\frac{d_{0}}{d_{i}}\right)^{\alpha}}}_{i=1} .
\end{aligned}
$$

$p_{\mathrm{r}}^{N}$ is the probability that the $\operatorname{SNR} \gamma_{N}:=R_{0} / N$ is above the threshold $\Theta$, i.e., the reception probability in a zero-interference network as it depends only on the noise. The second factor $p_{\mathrm{r}}^{I}$ is the reception probability in a zero-noise network.

This allows an independent analysis of noise and interference. If the load is light, then the number of interferers $k$ is relatively small and/or their distances are relatively big, which implies SIR $\gg$ SNR, thus the noise analysis alone provides accurate results. This has been demonstrated in [18] in the case of slotted ALOHA with transmit probability $p$. Indeed, for small $p$, the throughput is proportional to $p$, indicating that there are no losses due to collisions, i.e., $p_{\mathrm{r}}^{I} \approx 1$ for all timeslots and all transmissions. For high load, a separate interference analysis has to be carried out to determine $p_{\mathrm{r}}^{I}$. Note that power scaling, i.e., scaling the transmit powers of all the nodes by the same factor, does not change the SIR ( $p_{\mathrm{r}}^{I}$ only depends on power ratios), but (slightly) increases the SINR. Since only $p_{\mathrm{r}}^{N}$ is affected by absolute energy levels, we will focus on zero-interference networks to identify energy-efficient routing strategies.

In a zero-interference network, the reception probability over a link of distance $d$ at a transmit power $P_{0}$, is given by $p_{\mathrm{r}}:=$ $\mathbb{P}\left[\gamma_{N} \geqslant \Theta\right]=\exp \left(-\left(\Theta N / P_{0} d^{-\alpha}\right)\right)$, therefore

$$
P_{0}=\frac{d^{\alpha} \Theta N}{-\ln p_{\mathrm{r}}} .
$$

Note that for high probabilities, the packet loss probability $1-p_{\mathrm{r}}$ is tightly upperbounded by the normalized mean NSR $\Theta N / \bar{R}_{0}=\Theta / \bar{\gamma}_{N}[19]$. Since $-\ln p_{\mathrm{r}} \approx 1-p_{\mathrm{r}}$, we can also say

\footnotetext{
${ }^{2}$ A similar calculation has been carried out in [16, Appendix] for a network with spreading gain and equal transmit powers for all nodes.
}

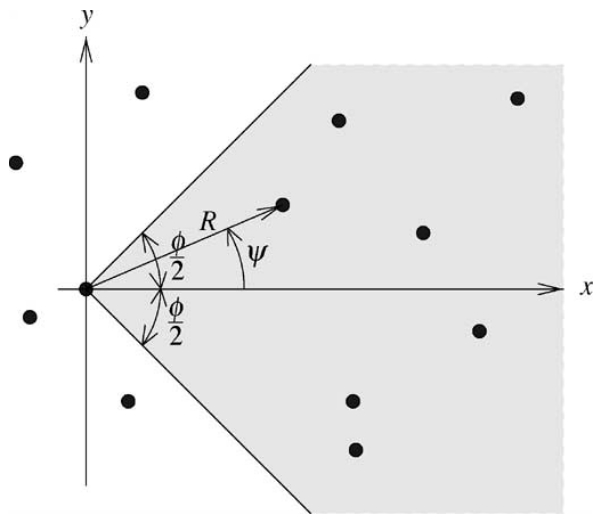

Fig. 1. Part of a Rayleigh network with the source at the origin and the $x$-axis pointing towards the destination node. $R$ denotes the distance to the nearest neighbor within a sector $\phi$ around $x$ and $\psi$ is its argument. Hence, $(R, \psi)$ are the polar coordinates of the nearest neighbor within a sector $\phi$ and $(X, Y)$ are its Cartesian coordinates.

that the packet loss probability is inversely proportional to the transmit power for high $p_{\mathrm{r}}$.

\section{B. Poisson Random Networks}

We consider a Poisson point process of intensity $\lambda$ in the plane, where the probability of finding $k$ nodes in an area $A$ is given by the Poisson distribution ${ }^{3}$

$$
\mathbb{P}[k \text { nodes in } A]=\mathrm{e}^{-\lambda A} \frac{(\lambda A)^{k}}{k !} .
$$

Note that for infinite networks, the Poisson point process corresponds to a uniform distribution [12], [20], and for large networks, the two distributions are equivalent for all practical purposes. Henceforth, we denote a network whose nodes constitute a two-dimensional Poisson point process as a Poisson random network. Without loss of generality, we will restrict ourselves to the case $\lambda=1$ (unit density), since the product $\lambda A$ can always be scaled such that $\lambda=1$.

For the routing schemes that we consider, we need to determine the distance from one node to its neighboring nodes that lie within a sector $\phi$, i.e., within $\pm \phi / 2$ of the source-destination axis (Fig. 1).

Proposition 1 (Distance to Nearest Neighbor): In a Poisson random network with unit density, the distance $R$ between a node and its nearest neighbor in a sector $\phi$ is Rayleigh distributed with mean $\sqrt{\pi /(2 \phi)}$.

Proof: Let $R$ be the distance to the nearest neighbor in a sector $\phi$. The probability that there is no neighbor in a sector $\phi$ up to a distance $r$ is the complementary cumulative distribution $\mathbb{P}[R>r]=\exp \left(-r^{2} \phi / 2\right)$, thus the probability density is $p_{R}(r)=r \phi \exp \left(-r^{2} \phi / 2\right)$, which is a Rayleigh distribution with mean $(\pi /(2 \phi))^{1 / 2}$ and variance $2 / \phi-\pi /(2 \phi)=(4-$ $\pi) /(2 \phi)$. The distribution of the argument $\psi$ is uniform between $-\phi / 2$ and $\phi / 2$.

\footnotetext{
${ }^{3}$ This can be generalized to higher dimensions if $A$ is the Lebesgue measure of the subset considered.
} 
Definition 1 (Rayleigh Network): A Rayleigh network is a Poisson random network where the physical channel is subject to Rayleigh fading.

To compare different routing schemes, we need to define the path efficiency.

Definition 2(Path Efficiency): The path efficiency is the ratio of the Euclidean distance between the end nodes and the actual distance traveled.

As an example, the path efficiency (for long connections ${ }^{4}$ ) in a square lattice network with nearest-neighbor routing is

$$
\kappa(\phi)=\frac{1}{|\cos \phi|+|\sin \phi|}
$$

where $\phi$ denotes the direction from transmitter to receiver relative to the orientation of the grid. The maximum is 1 , the minimum is $1 / \sqrt{2}$ (at $\phi=\pi / 4+k \pi / 2$ ), and the expected value is

$$
\eta:=\mathbb{E}[\kappa]=\frac{2 \sqrt{2}}{\pi} \operatorname{arctanh} \frac{\sqrt{2}}{2} \approx 0.79 .
$$

We will use $\eta$ to denote the expected path efficiency.

Definition 3 (Link Efficiency): The link efficiency is $X / R=$ $\cos (\Psi)$, where $X$ is the $x$-coordinate of the position of the nearest neighbor (see Fig. 1).

In Rayleigh networks with long connections, the distances and angles to nearest neighbors are independent identically distributed, so the expected path efficiency equals the expected link efficiency.

Proposition 2 (Path Efficiency in Rayleigh Networks): In a Rayleigh network with nearest-neighbor routing in a sector $\phi$, the expected path efficiency for a long connection is

$$
\eta(\phi)=\frac{2}{\phi} \sin \left(\frac{\phi}{2}\right) \approx 1-\frac{\phi^{2}}{24}
$$

where the approximation is the second-order Taylor expansion.

Proof: The expected link efficiency is $\mathbb{E}[\cos \Psi]$, and since the hop distances are i.i.d., this is also the expected path efficiency. Thus, we have

$$
\eta(\phi)=\mathbb{E}[\cos \Psi]=\frac{2}{\phi} \int_{0}^{\frac{\phi}{2}} \cos \psi \mathrm{d} \psi=\frac{2}{\phi} \sin \left(\frac{\phi}{2}\right) .
$$

To compare the transport capacity of different routing schemes, we have to determine the progress $X$. Changing to Cartesian coordinates, the joint probability $p_{X Y}(x, y)$ for the nearest neighbor's position (in a sector $\phi \leqslant \pi$ ) is

$$
\begin{aligned}
p_{X Y}(x, y)= & \mathrm{e}^{-\frac{x^{2}+y^{2}}{2} \phi} \\
& x \geqslant 0,-x \tan \left(\frac{\phi}{2}\right) \leqslant y \leqslant x \tan \left(\frac{\phi}{2}\right) .
\end{aligned}
$$

\footnotetext{
${ }^{4}$ This is based on the assumption that the angle towards the destination is uniformly distributed over $[0,2 \pi)$.
}

Integration with respect to $y$ yields the marginal density

$$
p_{X}(x)=\operatorname{erf}\left[\sqrt{\frac{\phi}{2}} \tan \left(\frac{\phi}{2}\right) x\right] \sqrt{\frac{2 \pi}{\phi}} \mathrm{e}^{-\frac{x^{2} \phi}{2}}
$$

where $\operatorname{erf}(\cdot)$ denotes the error function, i.e., $\operatorname{erf}(x):=$ $2 / \sqrt{\pi} \int_{0}^{x} \exp \left(-t^{2}\right) \mathrm{d} t$. It is easily verified that for small $\phi$, as $X \approx R$, this tends to a Rayleigh distribution. On the other hand, for $\phi \rightarrow \pi, \tan (\phi / 2) \rightarrow \infty$, the error function tends to 1 , and the distribution approximates the one-sided Gaussian $\sqrt{2} \exp \left(-x^{2} \pi / 2\right), x \geqslant 0$, with mean $\sqrt{2} / \pi$. $Y$ has zero mean and becomes Gaussian for $\phi \rightarrow \pi$ with variance $1 / \pi$. Note that $X$ and $Y$ are not independent unless $\phi=\pi$ or $\phi=2 \pi$.

The expected progress $\mathbb{E}[X]$ is

$$
\mathbb{E}[X]=\sqrt{\frac{\pi}{2 \phi}} \frac{2}{\phi} \sin \left(\frac{\phi}{2}\right)
$$

which corresponds, as expected, to the product of the mean path efficiency $\eta(\phi)(10)$ and the mean of the Rayleigh distribution $\sqrt{\pi /(2 \phi)}$.

Next, we generalize Proposition 1.

Proposition 3 (Distance to nth Neighbor): The probability density of the distance to the $n$th neighbor in a sector $\phi$ is

$$
p_{R_{n}}(r)=r^{2 n-1}\left(\frac{\phi}{2}\right)^{n} \frac{2}{(n-1) !} \mathrm{e}^{-\frac{r^{2} \phi}{2}} .
$$

Proof: Let $S_{k}$ be the $k$ th coefficient in the Poisson distribution: $S_{k}:=\left(r^{2} \phi / 2\right)^{k} / k !$. The probability that there are less than $n$ nodes closer than $r$ in the sector $\phi$ is

$$
P_{n}:=\mathbb{P}[0 \ldots n-1 \text { nodes within } r]=\sum_{k=0}^{n-1} S_{k} \mathrm{e}^{-\frac{r^{2} \phi}{2}} .
$$

Hence,

$$
\begin{aligned}
p_{R_{n}} & =\frac{\mathrm{d}}{\mathrm{d} r}\left(1-P_{n}\right) \\
& =(r \phi \sum_{k=0}^{n-1} S_{k}-\sum_{k=1}^{n-1} \underbrace{\frac{k\left(\frac{r^{2} \phi}{2}\right)^{k-1}}{k !}}_{S_{k-1}} r \phi) \mathrm{e}^{-\frac{r^{2} \phi}{2}} .
\end{aligned}
$$

The only term that is not cancelled in the two sums is the one at $n-1$, leading to

$$
p_{R_{n}}=r \phi \cdot \underbrace{S_{n-1} \mathrm{e}^{-\frac{r^{2} \phi}{2}}}_{\text {Erlang distribution }}
$$

which is identical to (15).

Since $p_{R_{n}}$ is a Rayleigh distribution for $n=1$, it can be considered a generalized Rayleigh distribution. Similarly, in one dimension, the Erlang distribution is a generalized exponential distribution. So, the transition from one dimension to two dimensions simply entails a multiplication by $r \phi$ (that comes from the inner derivative of the exponential part) in the distributions of the node distances. 
The mean of $R_{n}$ is given by

$$
\mathbb{E}\left[R_{n}\right]=\frac{\sqrt{2}}{\sqrt{\phi}} \frac{\Gamma\left(n+\frac{1}{2}\right)}{\Gamma(n)} \approx \frac{\sqrt{2}}{\sqrt{\phi}} \sqrt{n-1+\frac{\pi}{4}} .
$$

To get this approximation, we take the first term from the series expansion $\Gamma(n+1 / 2) / \Gamma(n)=\sqrt{n}(1-(1 / 8 n)+$ $\left.O\left(1 / n^{2}\right)\right)[21]^{5}$ and, noting that this is not accurate for small $n$, adjust it (by adding $\pi / 4-1$ to $n$ ) such that the approximation is precise for $n=1$. For $n>1$, this yields a very tight upper bound.

The second moment is $2 n / \phi$, hence, the variance is

$$
\operatorname{Var}\left[R_{n}\right]=\frac{2 n}{\phi}-E\left[R_{n}\right]^{2} \approx \frac{4-\pi}{2 \phi}
$$

using the same approximation. From the series expansion above, we know that $\operatorname{Var}\left[R_{n}\right]=(1 / 2 \phi)+O(1 / n)$. So we have found sharp lower and upper bounds on the variance

$$
\frac{4-\pi}{2 \phi} \leqslant \operatorname{Var}\left[R_{n}\right]<\frac{1}{2 \phi} \quad \forall n \in \mathbb{N} .
$$

Since $4-\pi \approx 1$, we can conclude that, interestingly, the variance is almost independent of $n .^{6}$

\section{Energy Consumption of a Route}

Assume a connection from node 0 to node $n$ in a multihop network. The desired end-to-end reliability is $P_{\mathrm{EE}}$. The reception probability of a chain of $n$ nodes is

$$
p_{n}=\prod_{i=1}^{n} \mathrm{e}^{-\frac{\Theta}{\gamma_{i}}}=\mathrm{e}^{-\Theta \sum_{i=1}^{n} \frac{1}{\bar{\gamma}_{i}}}
$$

where $\bar{\gamma}_{i}$ denotes the mean SNR at link $i$.

Assume that the maximum transmit power $P_{\max }$ is sufficient to reach node $n$ in a single hop with probability of $P_{\mathrm{EE}}$. The question is how many hops are optimum in terms of energy consumption. First, we consider the case of a one-dimensional chain of equidistant nodes with distance $d$. Let $E_{0}$ be the energy required for a transmission over distance $d$ with probability $P_{\mathrm{EE}}$, i.e., $E_{0}:=-d^{\alpha} \Theta N / \ln P_{\mathrm{EE}}$. Covering the total distance in one single hop requires an energy of $E_{1}=n^{\alpha} E_{0}$. In the multihop case with $n$ hops, a reception probability $p_{\mathrm{r}}=\sqrt[n]{P_{\mathrm{EE}}}$ is required at each hop. Since $\ln P_{\mathrm{EE}}=n \ln p_{\mathrm{r}}$, the total energy in this case is $E_{n}=n \cdot n E_{0}$. So, for $\alpha=2$, there is no benefit in multihop routing.

For a regular rectangular lattice network where the maximum-hop case corresponds to routing over nearest neighbors, we still have $E_{n}=n^{2} E_{0}$, whereas for the single-hop

\footnotetext{
${ }^{5}$ Note that this series can also be derived by using the identity $\Gamma(n+$ $1 / 2) / \Gamma(n)=[(2 n) ! \sqrt{\pi}] /\left[n !(n-1) ! 4^{n}\right] \quad[22]$ and applying Stirling's approximation.

${ }^{6}$ This is not the case for one-dimensional networks, where the variance increases linearly in $n$ (variance of the Erlang distribution with parameter $n$ ).
}

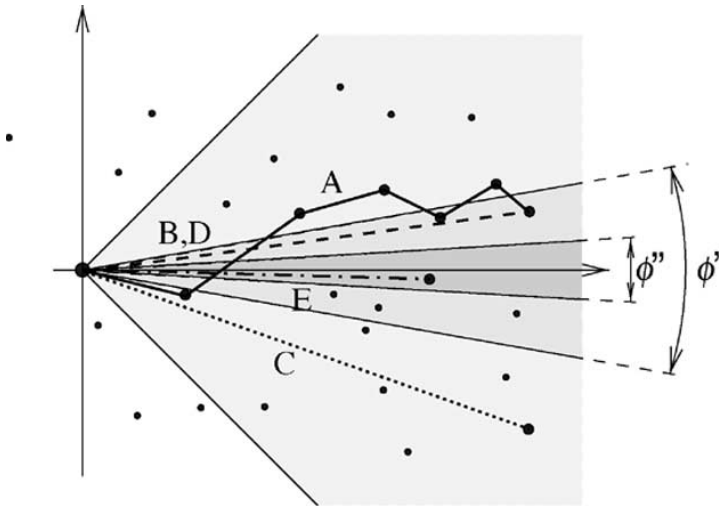

Fig. 2. Illustration of the different routing strategies. Strategy A follows the solid line (multiple hops), B and D follow the dashed line, C follows the dotted line, and $\mathrm{E}$ follows the dash-dotted line. The sectors $\phi, \phi^{\prime}$, and $\phi^{\prime \prime}$ are represented by different grades of gray.

case, we get $E_{1}=(n \kappa)^{\alpha} E_{0}$, where $\kappa$ is the path efficiency. The energy consumption is identical for

$$
\alpha=\frac{2 \log n}{\log (n \kappa)} .
$$

For $\alpha=4$ and $\kappa=1 / \sqrt{2}$, we get $E_{1}=E_{2}$, so even for large path loss exponents, there is no benefit in dividing a one-hop diagonal connection into two nearest-neighbor hops.

\section{Routing Strategies}

Here, we introduce the five routing strategies that will be analyzed in the next section. In order to implement these routing algorithms, it is assumed that all nodes know their own location and that the source node knows the direction towards the destination. The strategies are the following (see Fig. 2).

A) Multihop: Transmit hop by hop over $n$ nearest-neighbor hops in a sector $\phi$, i.e., the next-hop node is the nearest neighbor that lies within $\pm \phi / 2$ of the axis to the destination.

B) Single hop: Transmit directly to the $n$th node in the route found in A.

C) Single hop: Transmit directly to the $n^{\prime}$ th nearest neighbor in the sector $\phi$.

D) Single hop: Transmit directly to the $n$th nearest neighbor in a sector $\phi^{\prime}<\phi$.

E) Single hop: Transmit directly to the nearest neighbor in a sector $\phi^{\prime \prime}<\phi^{\prime}$.

Note that "single hop" here does not mean that the (final) destination is reached in one hop, but it means that a certain progress $X_{0}$ is made in one hop rather than in $n$ hops. So, relative to the end-to-end connection, all the schemes are multihop, but A uses short hops, whereas B through E use long(er) hops. For a fair comparison, we shall choose $n^{\prime}, \phi^{\prime}$, and $\phi^{\prime \prime}$ for strategies C, D, and $\mathrm{E}$, respectively, such that the expected progress $\bar{X}$ is the same as for A and B. As long as routing decisions are taken locally, virtually all routing strategies fall into those categories; whatever the specific properties of an algorithm are, the goal will always be to make some progress in the right direction. 


\section{ENERGy CONSUMPTION}

In this section, the energies required to deliver a packet over a progress $\bar{X}:=n \eta(\phi) \mathbb{E}[R(\phi)]=n \eta(\phi) \sqrt{\pi /(2 \phi)}$ with probability $P_{\mathrm{EE}}$ are determined for those five strategies, normalized by $\Theta N /\left(-\ln P_{\mathrm{EE}}\right)$.

\section{A. Analysis of the Five Routing Strategies}

Strategy A: With $p_{\mathrm{r}}=\sqrt[n]{P_{\mathrm{EE}}}$, the total expected energy consumption is $n^{2} \mathbb{E}\left[R^{\alpha}\right]$

$$
E_{\mathrm{A}}=n^{2} \underbrace{\left(\frac{2}{\phi}\right)^{\frac{\alpha}{2}} \Gamma\left(1+\frac{\alpha}{2}\right)}_{\mathbb{E}\left[R^{\alpha}\right]} .
$$

Strategy B: First, we need to establish the path efficiency. In strategy A, the angle to the next node is uniformly distributed in $\pm \phi / 2$. With increasing $n$, the argument $\Psi$ from the origin to the $n$th node tends to be Gaussian distributed with variance $V(\phi, n)$. Since the support of the probability density function of $\Psi$ is always $[-\phi / 2, \phi / 2],{ }^{7}$ the variance decreases inversely proportional to $n$, i.e., $V(\phi, n) \approx \phi^{2} / 12 n$. So, for large $n$, we get $^{8}$

$$
\begin{aligned}
\eta_{B}(\phi, n) & =\mathbb{E}[\cos \Psi] \\
& \approx \frac{\sqrt{6 n}}{\phi \sqrt{\pi}} \cdot \int_{-\infty}^{\infty} \cos \psi \mathrm{e}^{-6 n\left(\frac{\psi}{\phi}\right)^{2}} \mathrm{~d} \psi \\
& =\mathrm{e}^{-\frac{\phi^{2}}{24 n}} \\
& \approx 1-\frac{\phi^{2}}{24 n}
\end{aligned}
$$

from which we find $1 / \eta_{\mathrm{B}}(n, \phi)^{\alpha} \approx 1+\left(\alpha \phi^{2} / 24 n\right)$. Clearly, $\eta_{\mathrm{B}}(n, \phi) \geqslant \eta(\phi)$ with equality only for $n=1$. Since we are transmitting over a distance $n R\left(\eta / \eta_{\mathrm{B}}\right)$, we get

$$
\begin{aligned}
E_{\mathrm{B}} & =n^{\alpha} \mathbb{E}\left[R^{\alpha}\right]\left(\frac{\eta}{\eta_{\mathrm{B}}}\right)^{\alpha} \\
& \approx n^{\alpha}\left(\frac{2}{\phi}\right)^{\frac{\alpha}{2}} \Gamma\left(1+\frac{\alpha}{2}\right)\left(1-\frac{\alpha \phi^{2}(n-1)}{24 n}\right) .
\end{aligned}
$$

So, the increase of $n^{\alpha-2}$ compared with strategy $\mathrm{A}$ is partially compensated for by the factor $\left(\eta / \eta_{\mathrm{B}}\right)^{\alpha}$, i.e., the increase in path efficiency.

Strategy C: A direct transmission to the $n^{\prime}$ th neighbor in the sector $\phi$ shall result in the same expected progress as in strategy A, i.e., $\mathbb{E}\left[R_{n^{\prime}}\right]=n \mathbb{E}[R]$. From (19), we have

$$
\frac{\sqrt{2}}{\sqrt{\phi}} \sqrt{n^{\prime}-1+\frac{\pi}{4}}=n \sqrt{\frac{\pi}{2 \phi}}
$$

\footnotetext{
${ }^{7}$ Hence, after every convolution, the support needs to be scaled, which results in a reduction of the variance.

${ }^{8}$ The Gaussian approximation is very accurate even for small $n$. For $\phi=$ $\pi / 2$ and $n=1$, e.g., the precise value is $2 \sqrt{2} / \pi \approx 0.9003$, whereas this approximation yields $e^{-\pi^{2} / 96} \approx 0.9023$, so the error is only $0.2 \%$. The second-order Taylor expansions are identical, even for $n=1$.
}

which yields

$$
n^{\prime}=\frac{\pi}{4}\left(n^{2}-1\right)+1
$$

independent of $\phi$. The path efficiency for this single-hop transmission is still $\eta(\phi)$, since the argument of the destination node is uniformly distributed in the sector $\phi$. We have ${ }^{9}$

$$
\begin{aligned}
\mathbb{E}\left[R_{n^{\prime}}^{\alpha}\right] & =\left(\frac{2}{\phi}\right)^{\frac{\alpha}{2}} \frac{\Gamma\left(n^{\prime}+\frac{\alpha}{2}\right)}{\Gamma\left(n^{\prime}\right)} \\
& \approx\left(\frac{2}{\phi}\right)^{\frac{\alpha}{2}}\left(n^{\prime}-1+\sqrt{\alpha} \frac{\pi}{4}\right)^{\frac{\alpha}{2}} .
\end{aligned}
$$

Plugging in (31) gives

$$
\mathbb{E}\left[R_{n^{\prime}}^{\alpha}\right] \approx\left(\frac{\pi}{2 \phi}\right)^{\frac{\alpha}{2}}\left(n^{2}+\sqrt{\alpha}-1\right)^{\frac{\alpha}{2}} .
$$

Strategy D: The reduction of the sector angle from $\phi$ to $\phi^{\prime}$ shall ensure that the transmission to the $n$th nearest neighbor within $\phi^{\prime}$ results in the same progress as $n$ nearest-neighbor hops in $\phi$, i.e.,

$$
n \mathbb{E}[R(\phi)] \cdot \eta(\phi)=\mathbb{E}\left[R_{n}\left(\phi^{\prime}\right)\right] \cdot \eta\left(\phi^{\prime}\right) .
$$

Since $R$ and $R_{n}$ depend on $\phi$ and $\phi^{\prime}$, respectively, $\phi^{\prime}$ cannot be expressed in a closed form. However, we can find a tight upper bound on the energy consumption (lower bound on $\phi^{\prime}$ ) by assuming that the path efficiency does not increase with decreasing angle

$$
\phi^{\prime} \gtrsim \phi \frac{4(n-1)+\pi}{n^{2} \pi} .
$$

$\phi^{\prime} \approx \phi / n$ with high accuracy. With (32), we get the upper bound $B_{\mathrm{D}}$

$$
\begin{aligned}
E_{\mathrm{D}} & :=\mathbb{E}\left[R_{n}^{\alpha}\right] \lesssim B_{\mathrm{D}} \\
& =\left(\frac{2 \pi n^{2}}{\phi[4(n-1)+\pi]}\right)^{\frac{\alpha}{2}} \frac{\Gamma\left(n+\frac{\alpha}{2}\right)}{\Gamma(n)} .
\end{aligned}
$$

To get a lower bound, we assume that the path efficiency for $\phi^{\prime}$ is 1. Solving $n \mathbb{E}[R(\phi)] \eta(\phi)=\mathbb{E}\left[R_{n}\left(\phi^{\prime}\right)\right]$ using (19) yields

$$
\phi^{\prime} \lesssim \phi \frac{4(n-1)+\pi}{n^{2} \pi} \frac{1}{\eta(\phi)^{2}}
$$

and we find

$$
B_{\mathrm{D}} \gtrsim E_{\mathrm{D}} \gtrsim B_{\mathrm{D}} \eta(\phi)^{\alpha} .
$$

For large $n, E_{\mathrm{D}}$ tends to the lower bound. Since $B_{\mathrm{D}}$ has the same asymptotic behavior for large $n$ as strategy C, strategy D outperforms C.

\footnotetext{
${ }^{9}$ Again, we use the series expansion $\Gamma\left(n^{\prime}+\alpha / 2\right) / \Gamma\left(n^{\prime}\right)=n^{\prime(\alpha / 2)}[1-$ $\left.O\left(1 / n^{\prime}\right)\right]$ and adjust the constant such that the approximation is precise for $\alpha=n^{\prime}=1$. Note that for $\alpha=1$, this is identical to (19). For $\alpha=2, \mathbb{E}\left[R_{n^{\prime}}^{\alpha}\right]$ $\propto n^{\prime}$, and for $\alpha=4, \mathbb{E}\left[R_{n^{\prime}}^{\alpha}\right] \propto n^{\prime 2}$. The absolute error in the approximation for $\alpha=2$ is $\pi \sqrt{2} / 4-1 \approx 0.11$, independent of $n^{\prime}$.
} 
TABLE I

Normalized Energy Consumption of the Five Routing Strategies. The Approximations are SeCond-Order Taylor Expansions

\begin{tabular}{c|c|c|c}
\hline Strategy & $\alpha=2$ & $\alpha=3$ & $\alpha=4$ \\
\hline \hline $\mathrm{A}$ & $\frac{4}{\pi} \frac{1}{\eta^{2}} \approx \frac{4}{\pi}\left(1+\frac{\phi^{2}}{12}\right)$ & $\frac{6}{\pi} \frac{1}{\eta^{3}} \frac{1}{n} \approx \frac{6}{n \pi}\left(1+\frac{\phi^{2}}{8}\right)$ & $\frac{32}{\pi^{2}} \frac{1}{\eta^{4}} \frac{1}{n^{2}} \approx \frac{32}{n^{2} \pi^{2}}\left(1+\frac{\phi^{2}}{6}\right)$ \\
\hline $\mathrm{B}$ & $\frac{4}{\pi} \frac{1}{\eta_{\mathrm{B}}^{2}} \approx \frac{4}{\pi}\left(1+\frac{\phi^{2}}{12 n}\right)$ & $\frac{6}{\pi} \frac{1}{\eta_{\mathrm{B}}^{3}} \approx \frac{6}{\pi}\left(1+\frac{\phi^{2}}{8 n}\right)$ & $\frac{32}{\pi^{2}} \frac{1}{\eta_{\mathrm{B}}^{4}} \approx \frac{32}{\pi^{2}}\left(1+\frac{\phi^{2}}{6 n}\right)$ \\
\hline $\mathrm{C}$ & $\left(1+\frac{\frac{4}{\pi}-1}{n^{2}}\right) \frac{1}{\eta^{2}}$ & $\approx\left(1+\frac{(\sqrt{3}-1)^{\frac{3}{2}}}{n^{2}}\right) \frac{1}{\eta^{3}}$ & $\left(1+\frac{\frac{12}{\pi}-2}{n^{2}}+\frac{\frac{32}{\pi^{2}}-\frac{12}{\pi}+1}{n^{4}}\right) \frac{1}{\eta^{4}}$ \\
\hline $\mathrm{D}$ & $\approx\left(1+\frac{\frac{4}{\pi}-1}{n}\right)\left(1+\frac{\phi^{2}}{12 n^{2}}\right)$ & $\approx\left(1+\frac{\frac{6}{\pi}-1}{n}\right)\left(1+\frac{\phi^{2}}{8 n^{2}}\right)$ & $\approx\left(1+\frac{\frac{32}{\pi^{2}}-1}{n}\right)\left(1+\frac{\phi^{2}}{6 n^{2}}\right)$ \\
\hline $\mathrm{E}$ & $\frac{4}{\pi} \frac{1}{\eta^{\prime \prime 2}} \approx \frac{4}{\pi}\left(1+\frac{\phi^{2}}{12 n^{4}}\right)$ & $\frac{6}{\pi} \frac{1}{\eta^{\prime \prime 3}} \approx \frac{6}{\pi}\left(1+\frac{\phi^{2}}{8 n^{4}}\right)$ & $\frac{32}{\pi^{2}} \frac{1}{\eta^{\prime \prime 4}} \approx \frac{32}{\pi^{2}}\left(1+\frac{\phi^{2}}{6 n^{4}}\right)$ \\
\hline \hline
\end{tabular}

Strategy E: Here, we determine an even smaller angle $\phi^{\prime \prime}$ such that the first neighbor within that sector has the same expected distance as $n$ hops in the original sector $\phi$. As in D, the equation $n \mathbb{E}[R(\phi)] \eta(\phi)=\mathbb{E}\left[R\left(\phi^{\prime \prime}\right)\right] \eta\left(\phi^{\prime \prime}\right)$ does not have a closed-form solution, and we have to bound $E_{\mathrm{E}}$. From $\eta\left(\phi^{\prime \prime}\right)>$ $\eta(\phi)$ we have $\phi^{\prime \prime} \gtrsim \phi / n^{2}$ and thus

$$
E_{\mathrm{E}}<B_{\mathrm{E}}:=n^{\alpha}\left(\frac{2}{\phi}\right)^{\frac{\alpha}{2}} \Gamma\left(1+\frac{\alpha}{2}\right) \text {. }
$$

On the other hand, assuming $\eta\left(\phi^{\prime \prime}\right)=1$, we find a tight (for $n>2$ ) lower bound $B_{\mathrm{E}} \eta(\phi)^{\alpha}$, hence,

$$
B_{\mathrm{E}}>E_{\mathrm{E}} \gtrsim B_{\mathrm{E}} \eta(\phi)^{\alpha} \text {. }
$$

\section{B. Comparison}

In the previous section, the energy was calculated for a transmission over a distance of $\bar{X}=n \mathbb{E}[R] \eta$. To determine the energy required per unit distance, the energies are divided by $\bar{X}^{\alpha}$. Table I compares the five proposed routing strategies.

\section{Remarks:}

1) For strategy A, the energy consumption decreases with increasing $n$ for $\alpha>2$. If we take end-to-end probabilities and path efficiencies into account, the benefit of multihop routing under Rayleigh fading is substantially smaller than under the deterministic "disk model": For $\alpha=2$, it is always detrimental to use short hops, and for $\alpha=3, \phi=\pi / 2$, and $n=2$, we have $E_{\mathrm{B}} / E_{\mathrm{A}} \approx 1.76$, so skipping one hop is only $76 \%$ more expensive, rather than $2^{\alpha-1}=400 \%$, as an overly simplistic analysis would yield (see the remarks in Section I).

2) The single-hop strategies B-E all have comparable performance. With increasing $n$, their energy consumption decreases since the path efficiency increases.

3) For strategy $\mathrm{C}$ and $\alpha=3$, the approximation does not precisely agree with $\mathrm{A}$ for $n=1$ due to the approximation of the $\Gamma(\cdot)$ function for half-integers.

4) For strategies B, D, and E, the path efficiencies tend to 1 with increasing $n$ at different speeds.

5) Strategy $D$ is the only one where the energy consumption goes to 1 with increasing $n$.

In terms of the complexity of the implementation of those schemes, B and D are similar to A, while E is simpler, since only one nearest neighbor has to be identified. $\mathrm{C}$, on the other hand, is impractical for large $n$ due to the increase in $n^{2}$ in (31). For $n=5$, for example, the 20th neighbor would need to be identified, which may cause too much overhead.

\section{Determination of the Sector Angle $\phi$}

From the energy analysis, it can be seen that it is desirable to choose $\phi$ as small as possible to achieve a high path efficiency. On the other hand, we have to make sure that a sufficient number of neighbors can be reached with high probability within the sector. The maximum distance $\hat{R}$ that can be covered at the peak transmit power $P_{\max }$ to guarantee a reception probability $p_{\mathrm{r}}$ is

$$
\hat{R}=\left(\frac{P_{\max }\left(-\ln p_{\mathrm{r}}\right)}{\Theta N}\right)^{\frac{1}{\alpha}}=\left[\frac{\gamma_{\max }}{\Theta}\left(-\ln p_{\mathrm{r}}\right)\right]^{\frac{1}{\alpha}} .
$$

If $p_{\mathrm{c}}$ is the desired probability of finding a neighbor within $\phi$, we get from Proposition 1

$$
\phi \geqslant \frac{-2 \ln \left(1-p_{\mathrm{c}}\right)}{\hat{R}^{2}}=-2 \ln \left(1-p_{\mathrm{c}}\right)\left(\frac{\Theta N}{P_{\max } \ln p_{\mathrm{r}}}\right)^{\frac{2}{\alpha}} .
$$

For $\phi=\pi / 2$, for example, the expected distance to the nearest neighbor is 1 , but for $p_{\mathrm{c}}=99.9 \%$ (high probability of connectivity), a range $\hat{R}=\sqrt{12 \ln 10 / \pi} \approx 3$ is needed.

\section{Delay Considerations}

Clearly, if the delay constraints are tight, routing over many short hops may be prohibitively slow. If, on the other hand, the delay induced by an $n$-hop connection is tolerable, the energy consumption in the single-hop case can be further reduced by exploiting time diversity in the form of retransmissions.

\section{A. Multiple Long-Hop Transmissions}

For a fair comparison, we assume a delay constraint of $n$ timeslots for both the multihop (over $n$ hops, i.e., one transmission in every slot) and the single-hop schemes.

We consider two strategies to exploit the fact that $n$ timeslots are available in the case of a single-hop transmission (over an $n$ times larger distance), namely the schemes with and without availability of channel state information (CSI) at the transmitter.

No CSI: Transmit $n$ Times: For a block fading channel, we can assume independent fading levels at each timeslot. Since the delay constraint gives time for (at most) $n$ transmissions, 

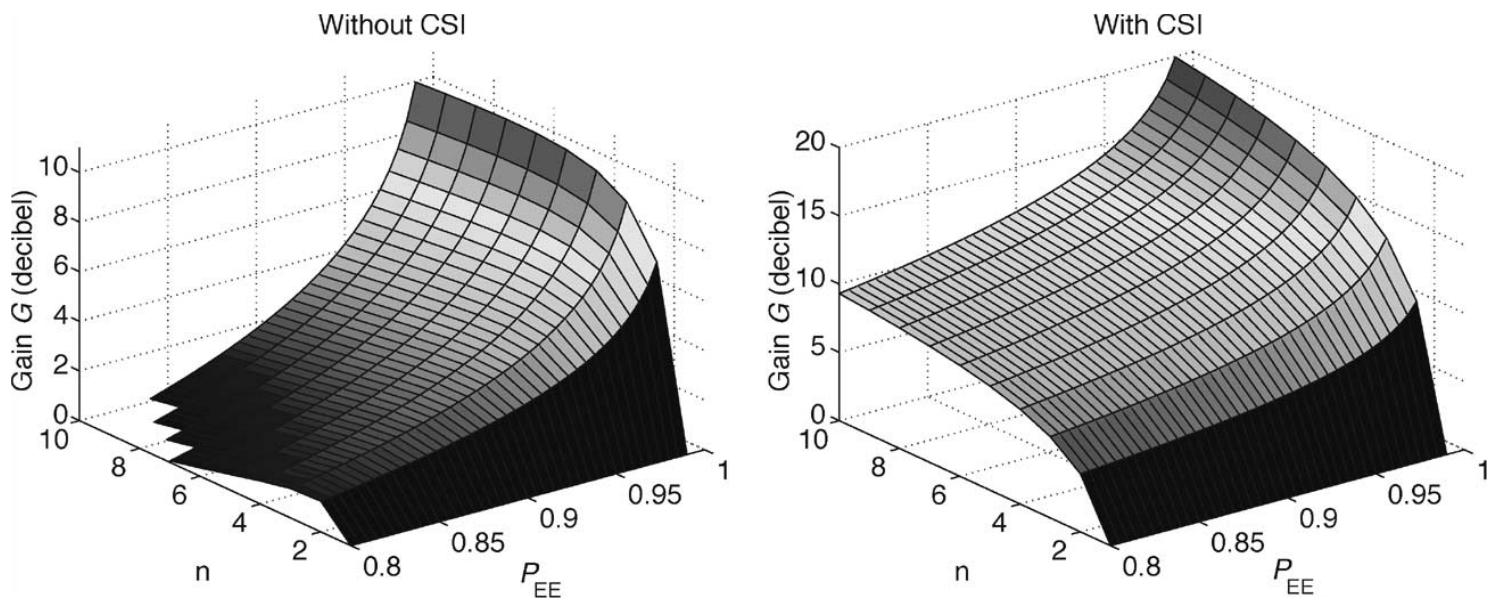

Fig. 3. Gain of the $n$-transmission schemes without (left) and with (right) channel state information over a single transmission in the first timeslot as a function of the number of available timeslots $n$ and the desired reliability $P_{\mathrm{EE}}$. Points with gain smaller than one are not shown.
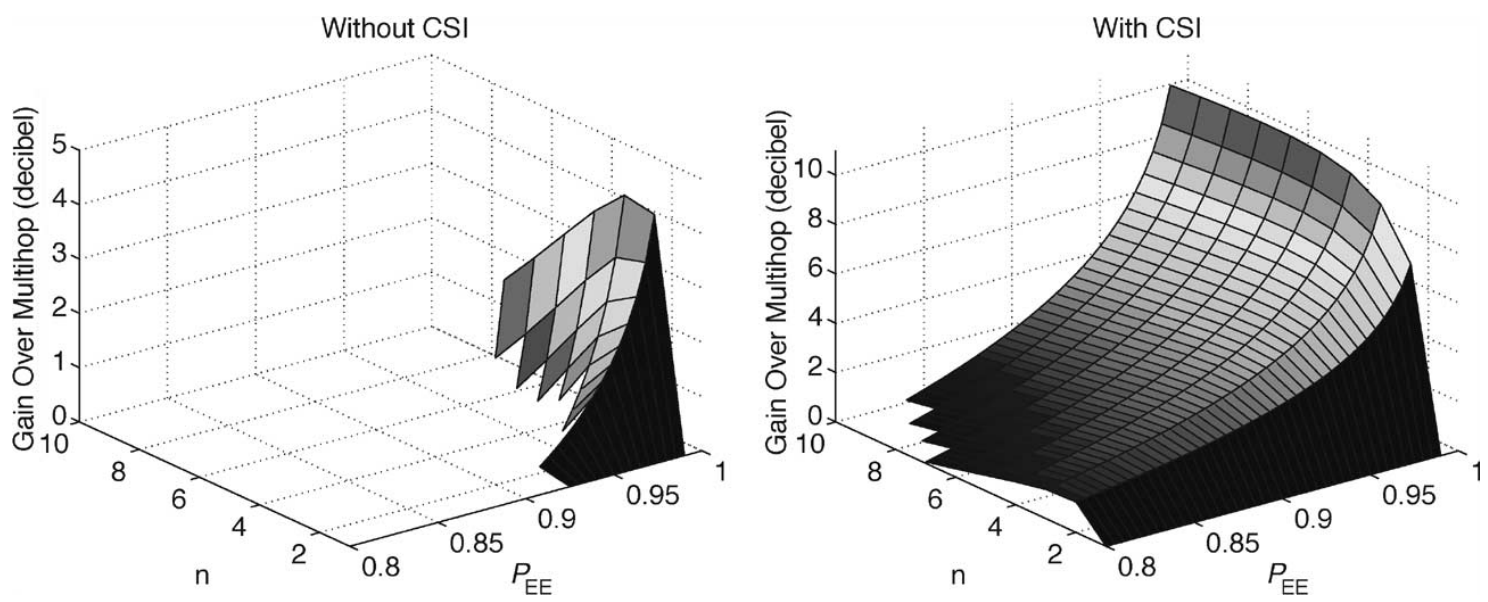

Fig. 4. Gain of the schemes without (left) and with (right) channel state information over a multihop scheme as a function of the number of available timeslots $n$ and the desired reliability $P_{\mathrm{EE}}$. The path loss exponent $\alpha$ is 3 . Points with gain smaller than one are not shown.

there is a benefit from time diversity (selection combining). The required single-use reception probability $P_{\mathrm{EE}, 1}$ is then given by

$$
P_{\mathrm{EE}, 1}=1-\left(1-P_{\mathrm{EE}}\right)^{\frac{1}{n}} .
$$

Compared with the single-transmission case, this leads to an energy gain of

$$
G=\frac{\log P_{\mathrm{EE}, 1}}{n \log P_{\mathrm{EE}}}
$$

since from (6), the necessary transmit power in the singletransmission case is $-\left(\Theta N d^{\alpha} / \log P_{\mathrm{EE}}\right)$, whereas for the multitransmission case, it is $-\left(n \Theta N d^{\alpha} / \log P_{\mathrm{EE}, 1}\right)$.

If we assume a simple automatic repeat request (ARQ) scheme, where a short ACK or NACK packet is sent immediately after the receipt of the data packet, this gain is almost doubled (and the average delay cut in half), since after a successful transmission, the packet does not have to be retransmitted any longer.

With CSI: A Single Opportunistic Transmission When the Channel is Best: With CSI at the transmitter, the packet can be scheduled to be transmitted when the fading level is best. In other words, the best timeslot out of $n$ is picked for a single transmission of the packet. Note that if the delay constraint $n$ is not hard but an average constraint, then this scheme may choose from $2 n$ slots. The cumulative distribution of the maximum of $n$ fading random variables is $F_{n}(x)=[1-\exp (-x / \bar{R})]^{n}$ with $\bar{R}=P_{0} d^{-\alpha}$. From

$$
P_{\mathrm{EE}}=1-\left(1-\mathrm{e}^{-\frac{\Theta N}{R}}\right)^{n} \Longrightarrow \bar{R}=\frac{\Theta N}{-\ln \left[1-\left(1-P_{\mathrm{EE}}\right)^{\frac{1}{n}}\right]}
$$

we see that the reception probability increases in the same way as in the multiple transmission case but with only one transmission. So, this strategy has an $n$ times higher gain than the one without CSI.

Fig. 3 shows the gains for both strategies (as a function of the number of transmissions $n$ and the desired probability) over a single-transmission scheme. For small $P_{\mathrm{EE}}$ and large $n$, the gain drops below 1 for the scheme without CSI (see the left plot).

Fig. 4 compares the single-hop retransmission-based strategies ( $n$ transmissions) with conventional multihop routing over 
$n$ hops for $\alpha=3$. It can be seen that the single-hop schemes often outperform multihop routing if the same delay is permitted. Since the factor $n$ between the strategies with and without CSI corresponds to an increase of the path loss exponent by one, we find in general

$$
G_{\mathrm{CSI}}(\alpha)=G_{\mathrm{NCSI}}(\alpha-1) \text {. }
$$

So, the left plot in Fig. 4 shows the gain $G_{\mathrm{CSI}}$ for $\alpha=4$.

Note that in this analysis, we did not take into account the higher path efficiency of the single-hop scheme. So, the effective gain is slightly bigger.

\section{B. Multiple Transmissions in the Short-Hop Case}

If the delay constraints are not tight, then there is time for retransmissions even for the short-hop routing scheme. Indeed, most wireless link layer protocols are based on ARQ, i.e., acknowledgments and retransmissions are employed to ensure a satisfactory reliability. A given desired link reliability $P_{\mathrm{L}}$ can be achieved by a single transmission or multiple transmissions at lower power. The following proposition addresses the question which scheme is energy optimum.

Proposition 4 (Optimum Power Level): The energyoptimum transmit power level $P_{0}^{\text {opt }}$ to achieve a link reliability $P_{\mathrm{L}}>1 / 2$ is given by

$$
P_{0}^{\mathrm{opt}}=\frac{\Theta N d^{\alpha}}{\ln 2}
$$

the corresponding single-transmission reception probability is $p_{\mathrm{r}}^{\mathrm{opt}}=1 / 2$, and the necessary average number of transmissions is ${ }^{10} n_{\mathrm{t}}=-\log _{2}\left(1-P_{\mathrm{L}}\right)$.

Proof: The average number of transmissions to achieve $P_{\mathrm{L}}$ is given by

$$
n_{\mathrm{t}}= \begin{cases}\frac{\log \left(1-P_{\mathrm{L}}\right)}{\log \left(1-p_{\mathrm{r}}\right)} & p_{\mathrm{r}}<P_{\mathrm{L}} \\ 1 & p_{\mathrm{r}} \geqslant P_{\mathrm{L}}\end{cases}
$$

So, the total energy consumption is

$$
E\left(P_{0}\right)=n_{\mathrm{t}} P_{0}=\frac{\log \left(1-P_{\mathrm{L}}\right)}{\log \left(1-\mathrm{e}^{-\frac{\Theta N}{P_{0} d^{-\alpha}}}\right)} P_{0}
$$

which is minimized at

$$
P_{0}^{\mathrm{opt}}=\underset{P_{0}}{\arg \min } E\left(P_{0}\right)=\frac{\Theta N d^{\alpha}}{\ln 2}
$$

corresponding to $p_{\mathrm{r}}^{\mathrm{opt}}=1 / 2$, and, in turn, $n_{\mathrm{t}}=-\log _{2}(1-$ $\left.P_{\mathrm{L}}\right)$.

Note that the optimum does not depend on $P_{\mathrm{L}}$. The factor $1 / \ln 2 \approx 1.44$ corresponds to $1.6 \mathrm{~dB}$, hence, the transmit power

${ }^{10} \log _{2}(\cdot)$ is the binary logarithm, while $\log (\cdot)$ denotes any logarithm. that minimizes the overall energy consumption is $1.6 \mathrm{~dB}$ above $\Theta N d^{\alpha}$. The minimum energy value is then given by

$$
E\left(P_{0}^{\mathrm{opt}}\right)=\Theta N d^{\alpha} \frac{-\ln \left(1-P_{\mathrm{L}}\right)}{(\ln 2)^{2}} .
$$

With $E\left(P_{0}^{\text {single }}\right)=-d^{\alpha} \Theta N / \ln P_{\mathrm{L}}$, the energy gain is

$$
\frac{E\left(P_{0}^{\text {opt }}\right)}{E\left(P_{0}^{\text {single }}\right)}=\log _{2}\left(1-P_{\mathrm{L}}\right) \log _{2} P_{\mathrm{L}}, \quad P_{\mathrm{L}}>\frac{1}{2} .
$$

The reduction in transmit power is $-\ln 2 / \ln P_{\mathrm{L}}$. For $P_{\mathrm{L}} \approx 1$, this is approximately $\ln 2 /\left(1-P_{\mathrm{L}}\right)$ or $\ln 2$ divided by the desired packet loss rate. The gain then is inversely proportional to the packet loss rate times the logarithm of the packet loss rate. For $P_{\mathrm{L}}=99 \%$, for example, the reduction in transmit power is $\approx 100 \ln 2 \approx 69$, the gain is slightly bigger than $10 \mathrm{~dB}$, and $n_{\mathrm{t}} \approx 6.64$. So, the reduction in transmit power more than compensates for the higher number of transmissions. Of course, the single-transmission scheme and the energyoptimum scheme are just the two extreme possibilities in the energy-delay tradeoff.

Again, to be fair, the long-hop scheme should be permitted the same delay. For the case without CSI, if the short-hop scheme routes over $n$ hops with an average of $n_{\mathrm{t}}$ transmissions at each hop, the long-hop scheme may use $n n_{\mathrm{t}}$ timeslots, i.e., it may transmit $n n_{\mathrm{t}}$ times instead of just $n$ times. The required reliability is $P_{\mathrm{EE}}=P_{\mathrm{L}}^{n}$, so we find that the energy consumption for $n$ transmissions is

$$
E_{n}=n \frac{\Theta N d^{\prime \alpha}}{-\ln \left[1-\left(1-P_{\mathrm{EE}}\right)^{\frac{1}{n}}\right]}
$$

where $d^{\prime}$ denotes the distance over this long hop. Permitting $n n_{\mathrm{t}}$ transmissions at lower transmit power, we have

$$
E_{n n_{\mathrm{t}}}=n n_{\mathrm{t}} \frac{\Theta N d^{\prime \alpha}}{-\ln \left[1-\left(1-P_{\mathrm{EE}}\right)^{\frac{1}{n n_{\mathrm{t}}}}\right]}
$$

which results in an energy gain of

$$
G=\frac{E_{n}}{E_{n n_{\mathrm{t}}}}=\frac{1}{n_{\mathrm{t}}} \frac{\log \left[1-\left(1-P_{\mathrm{EE}}\right)^{\frac{1}{n n_{\mathrm{t}}}}\right]}{\log \left[1-\left(1-P_{\mathrm{EE}}\right)^{\frac{1}{n}}\right]} .
$$

Since we know that the optimum single-transmission reception probability is $1 / 2$, this gain is smaller than 1 if $1-(1-$ $\left.P_{\mathrm{EE}}\right)^{1 / n} \leqslant 1 / 2$. Therefore, only for high $P_{\mathrm{EE}}$ or small $n$ is there a benefit in transmitting $n n_{\mathrm{t}}$ times. In contrast, the scheme with CSI available at the transmitter always benefits from a larger number of timeslots.

In summary, for very loose delay constraints, short-hop schemes derive more benefit from retransmissions. Since there is an optimum number of transmissions, the long-hop scheme cannot benefit from having a very large number of timeslots available with this simple retransmission scheme (unless CSI is available). In this case, the available time should rather be 
exploited by using a stronger channel code, thereby lowering the SINR threshold $\Theta$.

\section{CONCLuding Remarks}

We have offered a fresh look at the routing problem in random wireless networks by taking into account Rayleigh fading, end-to-end packet delivery probabilities, node distributions, and path efficiencies. The main result is that, from an energy point of view, routing over many short hops is not as beneficial in a Rayleigh network as it seems to be for the deterministic "disk model." If we impose a delay constraint that is, for a fair comparison, loose enough such that it can be met by short-hop routing, long-hop schemes may actually save energy.

Apart from energy and delay, there are other important practical issues that determine the overall performance of a routing scheme.

1) Interference. The different transmit power levels of the different routing schemes will change the interference by which other ongoing transmissions will be affected. However, if all nodes use a long-hop strategy rather than a short-hop strategy, their relative power levels will stay the same, and the interference part in the reception probability (5) $p_{\mathrm{r}}^{I}$ remains constant. Even if an individual source node chooses to adopt a long-hop strategy, the total transmission time will be shorter, which may offset the increase in power. In general, it is not clear whether multiple short hops or a single long hop causes more interference [10]. Total radiated energy may be an indicator.

2) Diversity schemes. Path and cooperative diversity have been shown to enhance the performance of wireless networks [19], [23]. In nearest-neighbor routing, there is little room to exploit cooperative strategies. If longer hops are used, a number of intermediate nodes may overhear the transmission and assist by relaying the packet if needed (provided that they are not asleep). In [4], the number of disjoint and independent paths are investigated. It has been shown that long-hop routing does not decrease the number of independent paths but increases the number of disjoint paths.

3) Receiver power consumption and sleep modes. The energy consumption for packet reception may be substantial. So, nodes that are not part of an active route (or cooperative scheme) should be put to sleep. Long-hop routing permits a more aggressive use of sleep modes than short-hop routing.

4) Amplifier characteristics. If the efficiency of the power amplifier depends on the radiated power, then the routing scheme should ensure that the PA mostly operates close to its saturated power $P_{\max }$ [24]. In this case, the variance of the distances becomes important, and nearest-neighbor routing should be avoided, as pointed out in [25].

5) Route acquisition time. Here, the long-hop schemes have a clear advantage, which is particularly important for ondemand routing algorithms [26].

6) Route breaks due to mobility and node failure. With less relaying nodes, the probability that new routes have to be discovered due to failing or moving nodes is smaller.
Hence, the robustness of the network is bigger. The availability of intermediate nodes also increases the network's capability of route repair and maintenance.

7) Energy and delay balancing. Often, the data gathered by sensor networks has to be delivered to a single point, the observer or base station. Using nearest-neighbor routing, the lifetime of a node close to the base station is much shorter than the lifetime of a node far away due to traffic accumulation [27]. With long-hop routing, the energy consumption is better balanced. Instead of just a few critical nodes, there are many more, which results in an increase of the network lifetime.

In conclusion, long-hop strategies have a clear advantage in most of these categories, in addition to being competitive in terms of energy.

\section{ACKNOWLEDGMENT}

The author would like to thank the anonymous reviewers for their helpful comments.

\section{REFERENCES}

[1] J. A. Silvester and L. Kleinrock, "On the capacity of multihop slotted ALOHA networks with regular structure," IEEE Trans. Commun., vol. COM-31, no. 8, pp. 974-982, Aug. 1983.

[2] H. Takagi and L. Kleinrock, "Optimal transmission ranges for randomly distributed packet radio terminals," IEEE Trans. Commun., vol. COM-32, no. 3, pp. 246-257, Mar. 1984.

[3] L. Hu, "Topology control for multihop packet networks," IEEE Trans. Commun., vol. 41, no. 10, pp. 1474-1481, Oct. 1993.

[4] J. L. Wang and J. A. Silvester, "Maximum number of independent paths and radio connectivity," IEEE Trans. Commun., vol. 41, no. 10, pp. 1482 1493 , Oct. 1993.

[5] M. Sanchez, P. Manzoni, and Z. Haas, "Determination of critical transmission range in ad-hoc networks," in Multiaccess, Mobility and Teletraffic Wireless Communications (MMT), Venice, Italy, 1999, pp. 293-304.

[6] P. Gupta and P. R. Kumar, "The capacity of wireless networks," IEEE Trans. Inf. Theory, vol. 46, no. 2, pp. 388-404, Mar. 2000.

[7] M. Grossglauser and D. Tse, "Mobility increases the capacity of ad-hoc wireless networks," in IEEE Information Communications (INFOCOM), Anchorage, AL, 2001, pp. 477-486.

[8] G. Németh, Z. R. Turányi, and A. Valkó, "Throughput of ideally routed wireless ad hoc networks," ACM Mobile Comp. Commun. Rev., vol. 5, no. 4, pp. 40-46, Oct. 2001.

[9] C. Schurgers, V. Tsiatsis, S. Ganeriwal, and M. Srivastava, "Optimizing sensor networks in the energy-latency-density design space," IEEE Trans. Mob. Comput., vol. 1, no. 1, pp. 70-80, Jan.-Mar. 2002.

[10] A. Ephremides, "Energy concerns in wireless networks," IEEE Wireless Commun., vol. 9, no. 4, pp. 48-59, Aug. 2002.

[11] A. J. Goldsmith and S. B. Wicker, "Design challenges for energyconstrained ad hoc wireless networks," IEEE Wireless Commun., vol. 9, no. 4, pp. 8-27, Aug. 2002.

[12] E. S. Sousa and J. A. Silvester, "Optimum transmission ranges in a directsequence spread-spectrum multihop packet radio network," IEEE J. Sel. Areas Commun., vol. 8, no. 5, pp. 762-771, Jun. 1990.

[13] D. A. Maltz, J. Broch, and D. B. Johnson, "Lessons from a full-scale multihop wireless ad hoc network testbed," IEEE Pers. Commun., vol. 8, no. 1, pp. 8-15, Feb. 2001.

[14] D. Ganesan, B. Krishnamachari, A. Woo, D. Culler, D. Estrin, and S. Wicker. (2002). An empirical study of epidemic algorithms in large scale multihop wireless networks. Intel Research Report IRB-TR-02003. [Online]. Available: http://www.intel-research.net/Publications/ Berkeley/050220021703_19.pdf

[15] T. J. Shepard. (1996, Aug.). "A channel access scheme for large dense packet radio networks," in Association Computing Machinery Special Interest Group Data Communication (ACM SIGCOMM), Stanford, CA, pp. 219-230. [Online]. Available: http://www.acm.org/sigcomm/ sigcomm96/papers/shepard.ps 
[16] M. Zorzi and S. Pupolin, "Optimum transmission ranges in multihop packet radio networks in the presence of fading," IEEE Trans. Commun., vol. 43, no. 7, pp. 2201-2205, Jul. 1995.

[17] Y. Y. Kim and S. Li, "Modeling multipath fading channel dynamics for packet data performance analysis," Wireless Networks, vol. 6, no. 6, pp. 481-492, Dec. 2000

[18] M. Haenggi, "Probabilistic analysis of a simple MAC scheme for ad hoc wireless networks," in IEEE Circuits and Systems Workshop Wireless Communications and Networking, Pasadena, CA, Sep. 2002.

[19] —-, "Analysis and design of diversity schemes for ad hoc wireless networks," IEEE J. Sel. Areas Commun., vol. 23, no. 1, pp. 19-27, Jan. 2005 [Online]. Available: http://www.nd.edu/ mhaenggi/pubs/jsac_adhoc.pdf

[20] R. Meester and R. Roy, Continuum Percolation. Cambridge, U.K.: Cambridge Univ. Press, 1996.

[21] R. L. Graham, D. E. Knuth, and O. Patashnik, Concrete Mathematics: A Foundation for Computer Science, 2nd ed. Reading, MA: AddisonWesley, 1994.

[22] G. Arfken, Mathematical Methods for Physicists, 3rd ed. Orlando, FL: Academic, 1985.

[23] J. N. Laneman, D. N. C. Tse, and G. W. Wornell, "Cooperative diversity in wireless networks: Efficient protocols and outage behavior," IEEE Trans. Inf. Theory, vol. 50, no. 12, pp. 3062-3080, Dec. 2004 [Online]. Available: http://www.nd.edu/ jnl/pubs/it2002.pdf

[24] S. C. Cripps, RF Power Amplifiers for Wireless Communications. Norwood, MA: Artech House, 1999. ISBN 0-89006-989-1.

[25] M. Haenggi, "Twelve reasons not to route over many short hops," in IEEE Vehicular Technology Conf. (VTC Fall), Los Angeles, CA, Sep. 2004. pp. 3130-3134 [Online]. Available: http://www.nd.edu/ mhaenggi/pubs/ vtc04.pdf
[26] E. M. Royer and C.-K. Toh, "A review of current routing protocols for ad-hoc mobile wireless networks," IEEE Pers. Commun., vol. 6, no. 2, pp. 46-55, Apr. 1999

[27] M. Haenggi, "Energy-balancing strategies for wireless sensor networks," in IEEE Int. Symp. Circuits and Systems (ISCAS), Bangkok, Thailand, May 2003, pp. 25-28 [Online]. Available: http://www.nd. edu/ mhaenggi/pubs/iscas03.pdf

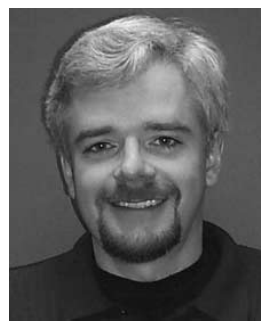

Martin Haenggi (S'95-M'99-SM'04) received the Dipl. Ing. (M.Sc.) degree in electrical engineering from the Swiss Federal Institute of Technology Zurich (ETHZ), Zurich, Switzerland, in 1995. In 1996, he earned the Dipl. NDS ETH (postdiploma) degree in information technology, and in 1999, he completed his Ph.D. thesis on the analysis, design, and optimization of cellular neural networks.

In 1995, he joined the Signal and Information Processing Laboratory at ETHZ as a Teaching and Research Assistant. After a postdoctoral year at the Electronics Research Laboratory at the University of California in Berkeley, he joined the faculty of the Electrical Engineering Department at the University of Notre Dame, Notre Dame, IN as an Assistant Professor in January 2001.

Dr. Haenggi was awarded the ETH medal for both his M.Sc. and Ph.D. theses and he received an NSF CAREER Award in 2005. He is a member of the Editorial Board of the Elsevier Journal on Ad Hoc Networks. His scientific interests include networking and wireless communications, with emphasis on ad hoc and sensor networks. 